\title{
Apatinib Enhances Chemosensitivity of FLT3-ITD Mutations acute Myeloid Leukemia Cells to Homoharringtonine Via VEGFR2 Pathway
}

\section{Yuanfei Shi}

Zhejiang University First Affiliated Hospital Department of Hematology

\section{Yan Zhang}

Huzhou Central Hospital

\section{Huafei Shen}

Zhejiang University First Affiliated Hospital Department of Hematology

\section{Xiujin Ye}

Zhejiang University First Affiliated Hospital Department of Hematology

Jie Jin

Zhejiang University First Affiliated Hospital Department of Hematology

wanzhuo xie ( $\nabla$ xiewanzhuo@zju.edu.cn )

Zhejiang University First Affiliated Hospital Department of Hematology

\section{Research Article}

Keywords: Apatinib, Homoharringtonine, Acute myeloid leukemia, VEGFR2, Anti-angiogenic agent, FLT3ITD mutations

Posted Date: March 10th, 2021

DOl: https://doi.org/10.21203/rs.3.rs-291831/v1

License: (c) (i) This work is licensed under a Creative Commons Attribution 4.0 International License. Read Full License 


\section{Abstract}

Purpose: The significance of vascular endothelial growth factor receptor (VEGFR)-3 in numerous solid tumors and acute myeloid leukemia (AML) has been demonstrated, but Apatinib remains largely unexplored. In this study, we explored whether Apatinib combined with HHT could kill AML cell lines and its possible mechanisms.

Methods: All cell lines were treated with Apatinib and HHT in different concentrations with control, Apatinib alone, HHT alone, and Apatinib combined with HHT. The changes of IC50 were measured by CCK8 assay, apoptosis rate, cell cycle, and mitochondrial membrane potential in each group were measured by flow cytometry. Finally, the possible cytotoxicity mechanism was analyzed by Western blotting.

Results: Our results noted that Apatinib combined with HHT remarkably inhibited cell proliferation, reduced the capacity of colony-forming, and induced apoptosis and cell cycle arrest in AML cells. Mechanistically, Apatinib and HHT play a role as a suppressor in the expression of VEGFR2 and the downstream signaling cascades, such as the PI3K, MAPK, and Stat3 pathways.

Conclusion: Our preclinical data demonstrate that Apatinib combined with HHT exerts a better antileukemia effect than Apatinib alone by inhibiting the VEGFR2 signaling pathway, suggesting the potential role for Apatinib and HHT in the treatment of AML.

\section{Introduction}

Acute myeloid leukemia (AML), derived from leukemia stem cells or progenitor cells. The American Cancer Society found that there are about 19,940 new cases of AML per annum with 11,180 deaths [1]. Although it has significantly improved the molecular biology of this disease and over the last forty years treatment has changed, the outcome remains poor for most patients [2]. Cytogenetic abnormalities are closely associated with clinical features and therapeutic responses in AML [3]. Therefore, there is a crying need for novel AML therapies, ranging from drugs targeting specific vascular endothelial growth factor (VEGF), oncogenic proteins to immunotherapies. In the last few years, a large number of studies have shown that angiogenesis is involved not only in leukemogenesis but also in leukemia progression [4]. Increased angiogenesis in the bone marrow niche relates to acute myeloid leukemia progression and resistance to treatment [5-9]. In consideration of the important role of angiogenic activity in different kinds of tumor-like hematological malignancies, targeting vasculogenesis signaling has won more and more attention as a new therapy to avoid cancer metastasis and resistance. Therefore, anti-angiogenic drugs or VEGFR inhibitors may provide a novel-innovative approach for AML treatments [10-12]. Apatinib (also known as YN968D1), a small molecule receptor tyrosine kinase inhibitor, can target VEGFR-2 selectively [13], which has been approved in China as a subsequent treatment for advanced gastric cancer [14]. Moreover, it has also been tested in phase II/III clinical trials of other cancers, including nonsmall cell lung cancer and breast cancer [15]. In this case, we are intrigued to explore whether Apatinib 
can be used for $A M L$ treatment and its relevant mechanisms. Homoharringtonine (HHT) (also known as omacetaxine mepesuccinate), a classical anti-leukemia drug, has been applied for about forty years in China. Although HHT has been used for the treatment of a variety of tumors, the specific targets are still unknown $[16,17]$. In this paper, we studied whether HHT would strengthen the anti-leukemic effect when combined with Apatinib. Besides, we also researched the underlying mechanisms of the cooperative effect of both two drugs.

\section{Materials And Methods Of Drugs}

Apatinib (Houston, TX, USA). HHT (Zhejiang Minsheng Pharmaceutical, Zhejiang, China) was dissolved in dimethyl sulfoxide (DMSO) at $1 \mathrm{mg} / \mathrm{mL}$ and stored at $-20^{\circ} \mathrm{C}$.

HHT was diluted with a culture medium in subsequent experiments.

\section{Cell culture}

MV4-11, MOLM-13, OCL-AML2, and OCL-AML3. Were purchased from the American Type Culture Collection (ATCC, Manassas, VA, USA). MV4-11 and MOLM13 expressed MLL fusion oncoprotein with FLT3-ITD. OCL-AML2 and OCL-AML3 expressed mutant NPM1c+. THP1 without FLT3-ITD or NPM1C+ mutant, all cells were cultured in RPMI 1640 with $20 \%$ FBS.

\section{Cell viability assay}

AML cell lines $\left(2 \times 10^{4}\right.$ cells/well) were plated in 96-well plates and then treated with different concentrations of Apatinib and HHT for $24 \mathrm{~h}$ and $48 \mathrm{~h}$. The cytotoxic effect was determined by cell counting kit-8 (CCK8; Dojindo, Japan) assay. IC50 (half-maximal inhibitory concentration) values were determined using a microplate reader (BIO-TEK EPOCH, USA).

\section{Apoptosis assay}

To assess apoptosis, MV4-11, MOLM-13, OCL-AML2, and OCL-AML3 cells were cultured and presented with different doses of Apatinib or HHT alone or in combination for $24 \mathrm{~h}$ or $48 \mathrm{~h}$ then double-labeled with Annexin-V-FITC/PI (eBioscience). However, the same concentration did not work in non-FLT3-ITD mutations AML cell lines, for example, THP1. The stained cells were analyzed with a NovoCyte flow cytometer (ACEA Biosciences, Inc.) with NovoExpress software.

Annexin V-positive cells were defined as apoptotic cells.

\section{In vitro clonogenicity assay}


MV4-11, MOLM-13, OCL-AML2, and OCL-AML3 cells ( $2 \times 10^{5} /$ well) were used to test colony-forming abilities. AML cells were seeded in 24-well plates and then, treated with $20 \mu \mathrm{M}$ Apatinib or $16 \mathrm{nM} \mathrm{HHT}$ alone or both molecules. After $24 \mathrm{~h}$, cells were washed and then cultured in complete methylcellulose medium at a cell density of 500 cells/well in 3.5-cm dishes for 10-14 days. The percentage of CFU was determined by counting colonies ( $\geq 50$ cells). Data were presented as the mean \pm S.D. of three independent experiments.

\section{Cell cycle analysis}

AML cells were treated with $24 \mathrm{~h}$, then cells were fixed overnight with $75 \%$ ethanol, PBS washes three times then incubation in buffer containing $50 \mu \mathrm{g} / \mathrm{mL}$ PI and $100 \mu \mathrm{g} / \mathrm{mL}$ RNase A for $30 \mathrm{~min}$ at room temperature. Cells were resuspended with DAPI saline solution and subjected to Flow Cytometer (NovoCyte ${ }^{\mathrm{TM}}$ ).

\section{Analysis of mitochondrial membrane potential}

AML cells $\left(2 \times 10^{5} / \mathrm{ml}\right)$ were plated in 24-well plates treated with various concentrations of Apatinib and $\mathrm{HHT}$. After $24 \mathrm{~h}$, cells were stained with $2 \mu \mathrm{M}$ Rhodamine 123 (Byeotime) for $30 \mathrm{~min}$. Then washed, mitochondrial membrane potential (MMP) was presented by flow cytometry (FACS Fortessa).

\section{Western Blot analysis}

Cell protein was extracted with RIPA protein lysis buffer (Beyotime). MOLM-13 cells

$\left(2 \times 10^{5} / \mathrm{mL}\right)$ were cultured with $20 \mu \mathrm{M}$ Apatinib or $16 \mathrm{nM} \mathrm{HHT}$ alone or combined for

$24 \mathrm{~h}$ and $48 \mathrm{~h}$. Related antibodies included $\beta$-actin, VEGFR2, p-VEGFR2, PI3K, Akt, CyclinA2, CyclinD1, P21, and Bcl-2 (rabbit, 1:1000, Cell Signaling Technology). Blots were tested by the addition of horseradish peroxidase (HRP)-conjugated secondary antibody. Signals were visualized using the ECL Western blotting detection kit (Gene-Flow, Staffordshire, UK).

\section{Statistical analysis}

We used GraphPad Prism software v7.0 to analyze the data. All experiments performed at least three independent experiments. Multi-group comparisons were using a one-way test of variance (ANOVA). Statistical analyses were presented using SPSS 20.0 software (La Jolla, CA).

\section{Results}




\section{The Cytotoxic Effect of Apatinib and HHT on FLT3-ITD mutations AML cell lines.}

We used the CCK8 assay to test the activity of Apatinib and HHT alone and in combination to verification the synergistic effect in inhibition of FLT3-ITD mutations AML cell viability. The concentrations of Apatinib and HHT were shown in Figure 1. We found that AML cell lines treated with both Apatinib and HHT showed a much better inhibitory effect especially in the FLT3-ITD group than those treated with each reagent alone in a time-dependent manner. The IC50 values for Apatinib and HHT were calculated used GraphPad Prism software v7.0 in AML cells, respectively (Table 1).

\section{Combination treatment with Apatinib and HHT plays a synergistic and lethal role in AML cell lines especially in FLT3-ITD mutations AML cells.}

To explore the synergistic effect of Apatinib and HHT on FLT3-ITD AML cells. Annexin V/PI staining was then performed to examine whether Apatinib would enhance HHT to induce apoptosis in FLT3-ITD AML cells. MV4-11, MOLM-13, OCL-AML2, and OCL-AML3 cells were exposed to the defined concentrations of Apatinib and HHT for $24 \mathrm{~h}$ or $48 \mathrm{~h}$. As shown in Figure 2A]2B, Apatinib or HHT alone was unable to induce apoptosis, while the combination could significantly increase apoptosis in all FLT3-ITD AML cell lines. Combination index $(\mathrm{Cl})$ values were calculated using the Chou and Talala software (Figure $2 \mathrm{C}$ ) and (Table 2). A Cl value of less than 1.0 means a synergistic effect. In contrast, the combination of Apatinib and HHT displayed no effect on the THP1 cell line without FLT3-ITD mutations (Figure 2D). These findings suggested that the combination of Apatinib and HHT might be a hopeful therapy for AML with FLT3-ITD mutation cells.

\section{The synergistic effects of Apatinib combined with HHT on the formation of colonies.}

Next, we researched the effects of Apatinib, HHT, or the combination of these two drugs on the cell colony formation in FLT3-ITD mutation cells. MV4-11, MOLM-13, OCL-AML2, and OCL-AML3 cells were treated with different concentrations of Apatinib or HHT alone or in combination for $48 \mathrm{~h}$. Neither Apatinib (20 $\mu \mathrm{M})$ nor HHT (16 nM) alone diminished the colony formation abilities on FLT3-ITD mutations AML cells. However, when Apatinib combined with $\mathrm{HHT}$, the colony-forming units significantly decreased $(\mathrm{P}<0.001$ vs. control, Apatinib alone, or HHT alone) (Figure 3).

Apatinib and HHT induces cell cycle arrest in FLT3-ITD mutations AML cells Cell cycle assays were carried out to investigate whether Apatinib combined with

HHT affects the cell cycle capacity in FLT3-ITD mutation cells. MV4-11 and MOLM-13 cells were treated with different concentrations of Apatinib alone or combined with HHT for 24 hours. As shown in Figure 4 A, cells treated with Apatinib at $20 \mu \mathrm{M}$ or $\mathrm{HHT}$ at $16 \mathrm{nM}$, these concentrations did not affect $\mathrm{G} 0 / \mathrm{G} 1$ or $\mathrm{S}$ phase cells notably. However, Apatinib and HHT exerted a prominent G0/G1 phase arrest and the S phase decreased. Then we used Western blots to explore the cycle-relevant proteins, such as Cyclin A2, Cyclin D1, and P21, the results were consistent with the former (Figure 4 B). Consistent with the cell cycle assays, cell cycle-relevant proteins of Apatinib plus HHT induced a reduction of Cyclin D1 and a rising of 

phase.

\section{Apatinib combined with HHT dose-dependent manner reduces the mitochondrial membrane potential}

We used the JC-1 probe to test the mitochondrial membrane potential (MMP, $\Delta \Psi \mathrm{m})$ to validate that the joint effects of Apatinib and HHT. As we expected, compared with each reagent alone, co-treatment with Apatinib and HHT remarkably reduced the MMP in MV4-11 and Molm13 cells after $24 \mathrm{~h}$ treatment (Figure 5A). Apatinib combined with HHT down-regulates the VEGFR2 and its downstream signaling pathways in AML cells. As we mentioned above, because Apatinib was a selective target to the VEGFR2 pathway. What surprises us was when Apatinib combined with HHT the VEGFR2 expression was affected. Apatinib and HHT treatment markedly inhibited the downstream signals, PI3K and p-Akt, as well as anti-apoptotic proteins like Bcl-2 and Mcl-1 (Figure 5B). We can summarize the mechanism through a brief schematic mechanism as follows: on FLT3-ITD mutations AML cells Apatinib combined with HHT induced cell apoptosis by decreasing mitochondrial membrane potential, inhibiting cell cycle and regulating vascular endothelial growth factor as well as its downstream signaling pathways (Figure $5 \mathrm{C}$ ).

\section{Discussion}

The evidence is overwhelming that the poor prognosis and higher disease relapse rate of AML accompanied with FLT3-ITD mutations make FLT3-ITD a perfect therapeutic target in individualized treatment [18-20]. Through our experimental results, we found that on FLT3-ITD (+) AML cell lines, compared to the monotherapy group Apatinib and HHT could significantly inhibit cell proliferation, promote cell apoptosis, and regulate Apatinib-relevant protein VEGFR2. However, these results were not found on the FLT3-ITD (-) THP1 cell line. So we proposed and proved for the first that the combination of Apatinib and HHT exerted a significant anti-leukemic action. Mechanistically, the combination of Apatinib and HHT synergistically decreases phosphorylated forms of VEGFR2 protein and its downstream PI3K, $\mathrm{Bcl}-2$, Akt, and Mcl-1, resulting in cell arresting at G1 and apoptosis. As we know, approximately $30 \%$ of AML with normal karyotype will have FLT3 (FMS-like tyrosine kinase 3) gene with mutations of internal tandem duplications (ITD) in the juxtamembrane domain [21]. Meanwhile, AML with FLT3-ITD mutations are concerned with poor overall survival (OS) and decreased disease-free survival (DFS) [22]. Although FLT3-mutant AML patients can be treated with FLT3 tyrosine kinase inhibitors (TKI), the relapse and rapid drug resistance limit its use [23]. Lately, several studies have found that HHT exerted a sensitive cytotoxic function on FLT3-ITD (+) AML cells [24].

The key role of angiogenesis, the process of forming blood vessels in the growth and maintenance of solid tumors. In the last few decades, a large number of studies show the involvement of angiogenesis in 
leukemogenesis as well as leukemia progression [25]. An increase in angiogenesis in the bone marrow niche is related to both acute lymphoblastic leukemia and acute myeloid leukemia [26-30]. Therefore, targeting angiogenesis with anti-angiogenic agents or VEGFR inhibitors is likely to a new method for AML treatment. Apatinib is a novel small-molecule tyrosine kinase inhibitor, which inhibits the phosphorylation of vascular endothelial growth factor receptor selectively, with a binding affinity ten times that of sorafenib. Besides, compared with the Apatinib sorafenib with only one-tenth of the anti-VEGFR-2 efficacy it is insufficient for anti-tumor angiogenesis. [31-33]. Its anti-tumor activity in all kinds of tumors has been proved in many studies [34-36]. Results of those clinical trials indicated the anti-tumor role of Apatinib across a large-scale of advanced cancers, but the specific function of tumor angiogenesis in AML pathogenesis remains unknown. Furthermore, our researches have found that Apatinib combined with a variety of anti-tumor drugs can improve the curative effect [37, 38]. It was interesting to note that accompanied by FLT3-ITD mutant AML cells presented a significant synergistic effect after treated with Apatinib and HHT. Nevertheless, it makes no difference to the AML cell line, which was without FLT3-ITD mutant (Fig. 2D). It has been well reported the important role of PI3K signaling in the progression of all kinds of tumors, including leukemia $[39,40]$. The evasion of apoptosis was an important characteristic of cancer, which was caused by the activation of anti-apoptotic molecules of the Bcl-2 protein family [4143]. Aberrant activation of $\mathrm{Bcl}-2$ members such as $\mathrm{Bcl}-2$ and $\mathrm{Mcl}-1$ were related to anti-apoptosis and drug resistance in FLT3-ITD mutant AML [44-46].

VEGF is considered a target in leukemia treatment and a variety of strategies have been applied to downregulate or inhibit the VEGF signaling pathway. The new strategy which inhibits VEGF signaling pathway could able to block the autocrine VEGF pathway in AML cells or the typical vessel development by the vascular endothelial cells [47]. We and other researchers found that Apatinib induced cytotoxicity was related to inhibition of the VEGFR2 and PI3K/Akt pathways, and induction of mitochondrial membrane protein (MMP)-mediated apoptosis. To our surprise, Apatinib and HHT could significantly enhance this phenomenon. As everyone knows that FLT3-ITD mutations result in missing auto-inhibitory function for FLT3 kinase, which next leads to activation of its downstream signaling pathways, such as PI3K/Akt and JAK/Stat5 [48-50]. Our results indicated that Apatinib combined with HHT synergistically suppressed the growth and induced apoptosis of FLT3-ITD mutations AML cells by synergistically down-regulating the expression of phosphorylated forms of VEGFR2 and PI3K/Akt signaling pathways as well as affecting the expression level of cell cycle regulatory protein, up-regulating the expressions of cyclin A2 and p21 and down-regulating the expression of cyclin D1 in FLT3- ITD (+) AML cell lines. Nevertheless, mechanisms of combined application of Apatinib and HHT in regulating P21, cyclin A2, and cyclin D1 proteins remain unclear and need further study.

\section{Conclusions}

All in all, our research first reveals the synergistic anti-leukemic effect between Apatinib and HHT on FLT3ITD mutant AML cells, likely through inhibiting VEGFR2-mediated signaling pathways, and suggests potential benefits and clinical application of Apatinib combined with HHT in the treatment of AML patients. 


\section{Declarations}

\section{Author contributions}

YS, YZ performed in vitro experiments and animal study. HFS carried out the statistical analysis. XJY and $\mathrm{JJ}$ designed the study. WZX wrote the manuscript. All authors read and approved the final manuscript.

\section{Compliance with Ethical Standards}

\section{Funding}

This work was supported in part by the Research Plan of the National Natural Science Foundation of China (No.81372256).

\section{Conflicts of interest}

The authors declare that they have no potential conflicts of interest.

\section{Ethical approval}

This article does not contain any studies with human participants or animals performed by any of the authors.

\section{Data Availability Statements}

Data sharing not applicable to this article as no datasets were generated or analysed during the current study.

\section{References}

1. Chen, H. S. et al. Predicting US- and state-level cancer counts for the current calendar year: Part I: evaluation of temporal projection methods for mortality. Cancer 118, 1091-1099, doi:10.1002/cancer.27404 (2012).

2. Perl, A. E. The role of targeted therapy in the management of patients with AML. Blood Adv 1, 22812294, doi:10.1182/blood advances.2017009829 (2017).

3. Walter, R.B. and E.H. Estey, Management of older or unfit patients with acute myeloid leukemia. Leukemia, 2015. 29(4): p. 770-5.

4. Kuykendall A, Duployez N, Boissel N, Lancet JE, Welch JS. Acute myeloid leukemia: the good, the bad, and the ugly. American Society of Clinical Oncology educational book. Am Soc Clin Oncol Annual Meeting. 2018; 38:555-73.

5. Shirzad R, Shahrabi S, Ahmadzadeh A, Kampen KR, Shahjahani M, Saki N. Signaling and molecular basis of bone marrow niche angiogenesis in leukemia. Clin Transl Oncol. 2016; 18:957-71. 
6. Todorovic M, Radisavljevic Z, Balint B, Andjelic B, Todorovic V, Jovanovic MP, et al. Increased angiogenesis-associated poor outcome in acute lymphoblastic leukemia: a single-center study. Appl Immunohistochem Mol Morphol. 2012; 20:488-93.

7. Kampen KR, Ter Elst A, de Bont ES. Vascular endothelial growth factor signaling in acute myeloid leukemia. Cell Mol Life Sci. 2013; 70:1307-17.

8. AbdEIAal Asmaa A, Afify RA, Zaher AE, ElGamal MM, Atef AM. Study of prognostic significance of marrow angiogenesis assessment in patients with de novaculite leukemia. Hematology. 2015; 20:504-10.

9. Karakurt N, Aksu T, Koksal Y, Yarali N, Tunc B, Uckan-Cetinkaya D, et al. Angiopoietins in the bone marrow microenvironment of acute lymphoblastic leukemia. Hematology. 2016; 21:325-31.

10. Passaro D, Di Tullio A, Abarrategi A, Rouault-Pierre K, Foster K, Ariza-McNaughton L, et al. Increased vascular permeability in the bone marrow microenvironment contributes to disease progression and drug response in acute myeloid leukemia. Cancer Cell. 2017; 32:324-41.

11. Chatterjee, L.C. Heukamp, M. Siobal, J. Schöttle, C. Wieczorek, M. Peifer, et al.TuMor VEGF: VEGFR2 autocrine feed-forward loop triggers angiogenesis in lung cancer, J. Clin. Investig. 123 (2013) 17321740.

12. Xie, J. Liu, H. Liu, S.H. Liang, M.G. Lin, Y.Y. Gu, et al., The antituMor effect of tanshinone IIA on antiproliferation and decreasing VEGF/VEGFR2 expression on the huMan non-small cell lung cancer A549 cell line, Acta Pharm. Sin. B 5 (2015) 554-563.

13. B.C. Brito, G.J. Lourenço, G.B. Oliveira, C.A. De Souza, J. Vassallo, C.S.P. Lima, Associations of VEGF and VEGFR2 polymorphisms with increased risk and aggressiveness of multiple myeloma, Ann. Hematol. 93 (2014) 1363-1369.

14. Tian S, Quan H, Xie C, Guo H, Lü F, Xu Y, et al. YN968D1 is a novel and selective inhibitor of vascular endothelial growth factor receptor-2 tyrosine kinase with potent activity in vitro and in vivo. Cancer Sci. 2011; 102:1374-80.

15. Li J, Qin S, Xu J, Guo W, Xiong J, Bai Y, et al. Apatinib for chemotherapy-refractory advanced metastatic gastric cancer: results from a randomized, placebo-controlled, parallel-arm, phase II trial. J Clin Oncol.2013; 31:3219-25.

16. Zhang H. Apatinib for molecular targeted therapy in the $t \mu$ Mor. Drug Des Dev Ther. $2015 ; 9: 6075-81$.

17. Lu S and Wang J (2014). Homoharringtonine and omacetaxine for myeloid hematological malignancies. J Hematol Oncol 7, 2.

18. Feldman E, Arlin Z, Ahmed T, Mittelman A, Puccio C, Chun H, Cook P, and Baskind P. Homoharringtonine is safe and effective for patients with acute myelogenous leukemia. Leukemia 6 , 1185-1188.

19. Thiede C, Steudel C, Mohr B, Schaich M, Schäkel U, Platzbecker U, et al. Analysis of FLT3-activating mutations in 979 patients with acute myelogenous leukemia: association with FAB subtypes and identification of subgroups with poor prognosis. Blood. 2002; 99:4326-35. 
20. Kuwatsuka Y, Tomizawa D, Kihara R, Nagata Y, Shiba N, et al. Prognostic value of genetic mutations in adolescent and young adults with acute myeloid leukemia. Int J Hematol. 2018; 107:201-10.

21. Ravandi F, Kantarjian H, Faderl S, Garcia-Manero G, O'Brien S, Koller C, et al. Outcome of patients with FLT3-mutated acute myeloid leukemia in first relapse. Leuk Res. 2010; 34:752-6.

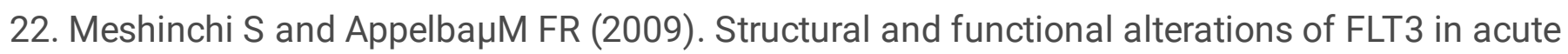
myeloid leukemia. Clin Cancer Res 15, 4263-4269.

23. Gilliland DG and Griffin JD (2002). The roles of FLT3 in hematopoiesis and leukemia. Blood 100, 1532-1542.

24. Kayser S and Levis MJ (2014). FLT3 tyrosine kinase inhibitors in acute myeloid leukemia: clinical implications and limitations. Leuk Lymphoma 55, 243-255.

25. Lam SS, Ho ES, He BL, et al (2016). Homoharringtonine (omacetaxine mepesuccinate) as an adjunct for FLT3-ITD acute myeloid leukemia. Sci Transl Med 8, 359ra129.

26. Shirzad R, Shahrabi S, Ahmadzadeh A, Kampen KR, Shahjahani M, Saki N. Signaling and molecular basis of bone marrow niche angiogenesis in leukemia. Clin Transl Oncol. 2016; 18:957-71.

27. Todorovic M, Radisavljevic Z, Balint B, Andjelic B, Todorovic V, Jovanovic MP, et al. Increased angiogenesis-associated poor outcome in acute lymphoblastic leukemia: a single-center study. Appl Immunohistochem Mol Morphol. 2012; 20:488-93.

28. Kampen KR, Ter Elst A, de Bont ES. Vascular endothelial growth factor signaling in acute myeloid leukemia. Cell Mol Life Sci. 2013; 70:1307-17.

29. AbdEIAal Asmaa A, Afify RA, Zaher AE, ElGamal MM, Atef AM. Study of prognostic significance of marrow angiogenesis assessment in patients with de novaculite leukemia. Hematology. 2015; 20:504-10.

30. Karakurt N, Aksu T, Koksal Y, Yarali N, Tunc B, Uckan-Cetinkaya D, et al. Angiopoietins in the bone marrow microenvironment of acute lymphoblastic leukemia. Hematology. 2016; 21:325-31.

31. Passaro D, Di Tullio A, Abarrategi A, Rouault-Pierre K, Foster K, Ariza- McNaughton L, et al. Increased vascular permeability in the bone marrow microenvironment contributes to disease progression and drug response in acute myeloid leukemia. Cancer Cell. 2017; 32:324-41.

32. Yang C, Qin S. Apatinib targets both tuMor and endothelial cells in hepatocellular carcinoma. Cancer Med. 2018; 7(9):4570-4583.

33. Tian S, Quan H, Xie C, et al. YN968D1 is a novel and selective inhibitor of vascular endothelial growth factor receptor-2 tyrosine kinase with potent activity in vitro and in vivo. Cancer Sci. 2011; 102(7):1374-1380.

34. Yingqiang Zhang, Wenzhe Fan et al. Apatinib for Patients With Sorafenib-Refractory Advanced Hepatitis B Virus Related Hepatocellular Carcinoma: Results of a Pilot Study.2019; 26: 1-8.

35. Zhang H. Apatinib for molecular targeted therapy in the tuMor. Drug Des Devel Ther 2015; 9:6075-81.

36. Liang S, Tong XZ, Fu LW. [Inhibitory effect of apatinib on HL-60 cell proliferation and its mechanism]. Nan Fang Yi Ke Da Xue Xue Bao 2011; 31:871-4. 
37. Qing Pan, Jiuhe Wang, Xianqin Jiang et al. Apatinib enhances chemosensitivity of acute myeloid leukemia hl60 cells to cytarabine by inducing apoptosis. JBUON 2019; 24(1): 375.

38. Tong XZ, Wang F, Liang $S$ et al. Apatinib (YN968D1) enhances the efficacy of conventional chemotherapeutical drugs in side population cells and ABCB1 overexpressing leukemia cells. Biochem Pharmacol 2012; 83:586-97.

39. Mi YJ, Liang YJ, Huang HB et al. Apatinib (YN968D1) reverses multidrug resistance by inhibiting the efflux function of multiple ATP-binding cassette transporters. Cancer Res 2010; 70:7981-91.

40. Pei, T. Si, Y. Lu, J.X. Zhou, L. Jiang, Salvianolic acid A, a novel PI3K/Akt inhibitor, induces cell apoptosis and suppresses t $\mu$ Mor growth in acute myeloid leukemia, Leukemia \& lymphoma, (2017) $1-9$.

41. Nepstad, H. Reikvam, A.K. Brenner, O. Bruserud, K.J. Hatfield, Resistance to the Antiproliferative In Vitro Effect of PI3K-Akt-mTOR Inhibition in Primary H $\mu$ Man Acute Myeloid Leukemia Cells Is Associated with Altered Cell Metabolism, Int J Mol Sci, 19 (2018).

42. Yip KW and Reed JC (2008). Bcl-2 family proteins and cancer. Oncogene 27, 6398-6406.

43. Hanahan D and Weinberg RA (2000). The hallmarks of cancer. Cell 100, 57-70.

44. Hanahan D and Weinberg RA (2011). Hallmarks of cancer: the next generation.Cell 144, 646-674.

45. Minami Y, Yamamoto K, Kiyoi H, Ueda R, Saito H, and Naoe T (2003). Different antiapoptotic pathways between the wild-type and mutated FLT3: insights into therapeutic targets in leukemia. Blood 102, 2969-2975.

46. Rahmani M, Aust MM, Hawkins E, et al (2015). Co-administration of the mTORC1/TORC2 inhibitor INK128 and the Bcl-2/Bcl-xL antagonist ABT-737 kills huMan myeloid leukemia cells through Mcl-1 down-regulation and AKT inactivation. Haematologica 100, 1553-1563.

47. Song G, Li Y, Jiang G (2012b). Role of VEGF/VEGFR in the pathogenesis of leukemias and as treatment targets (Review). Oncol Rep, 28, 1935-44.

48. Song G, Li Y, Jiang G (2012a). Role of VEGF/VEGFR in the pathogenesis of leukemias and as treatment targets (Review). Oncol Rep, 28, 1935-44.

49. Takahashi S (2011). Downstream molecular pathways of FLT3 in the pathogenesis of acute myeloid leukemia: biology and therapeutic implications. J Hematol Oncol 4, 13.

50. Steelman LS, Abrams SL, Whelan J, et al (2008). Contributions of the Raf/ MEK/ERK, $\mathrm{PI3K} / \mathrm{PTEN} / A k t / m T O R$, and Jak/STAT pathways to leukemia. Leukemia 22, 686-707.

\section{Figures}



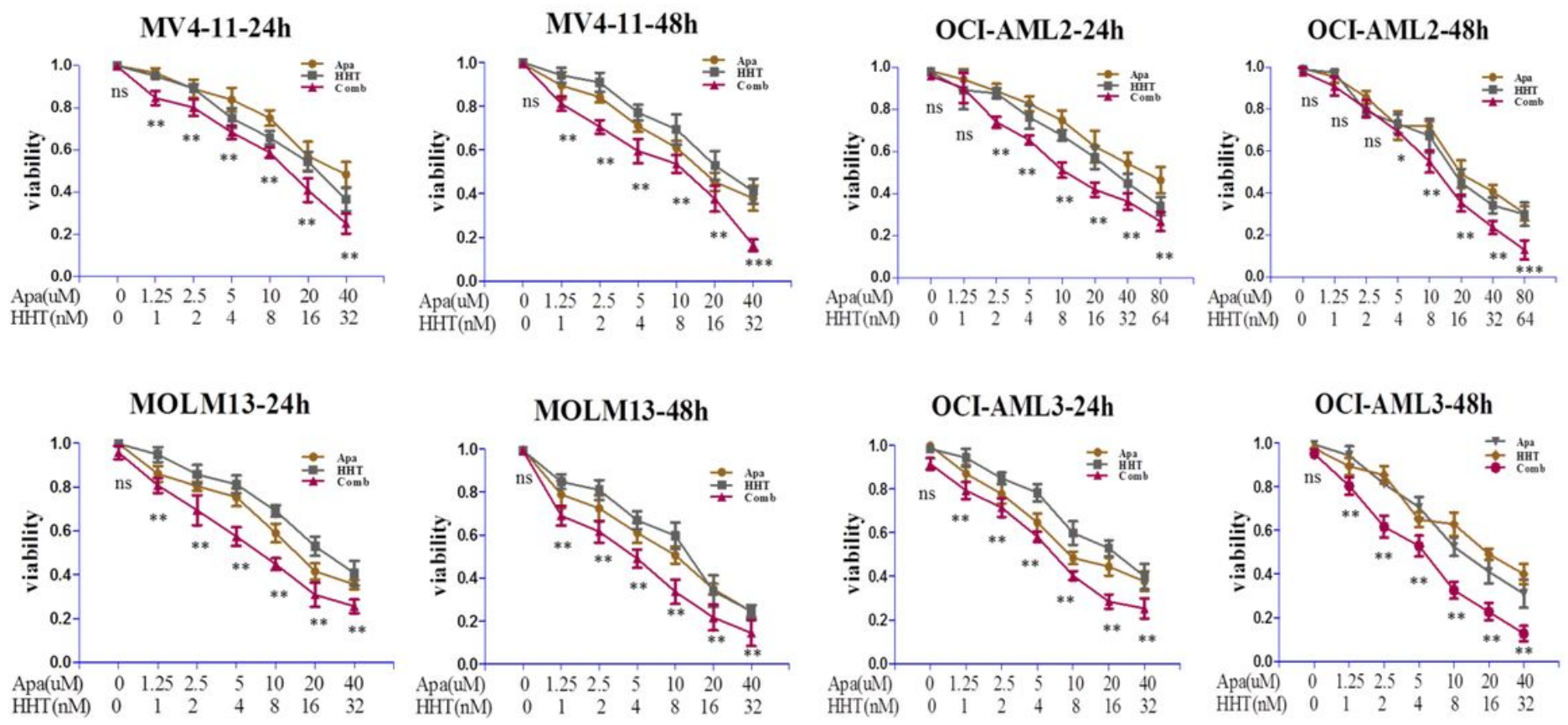

\section{Figure 1}

Cell viability after treatment with various doses of Apatinib or HHT alone or in combination for $24 \mathrm{~h}$ or 48 $\mathrm{h}$. The percent viability is normalized to the percent viability of the DMSO-treated control. Values are expressed as the mean \pm S.D. of three independent experiments. The half-maximal inhibitory concentration (IC50) values of Apatinib and HHT in a time-dependent manner on FLT3-ITD mutations AML cells. ${ }^{*} p<0.05 ;{ }^{* *} p<0.01 ; * \star * p<0.001$. 

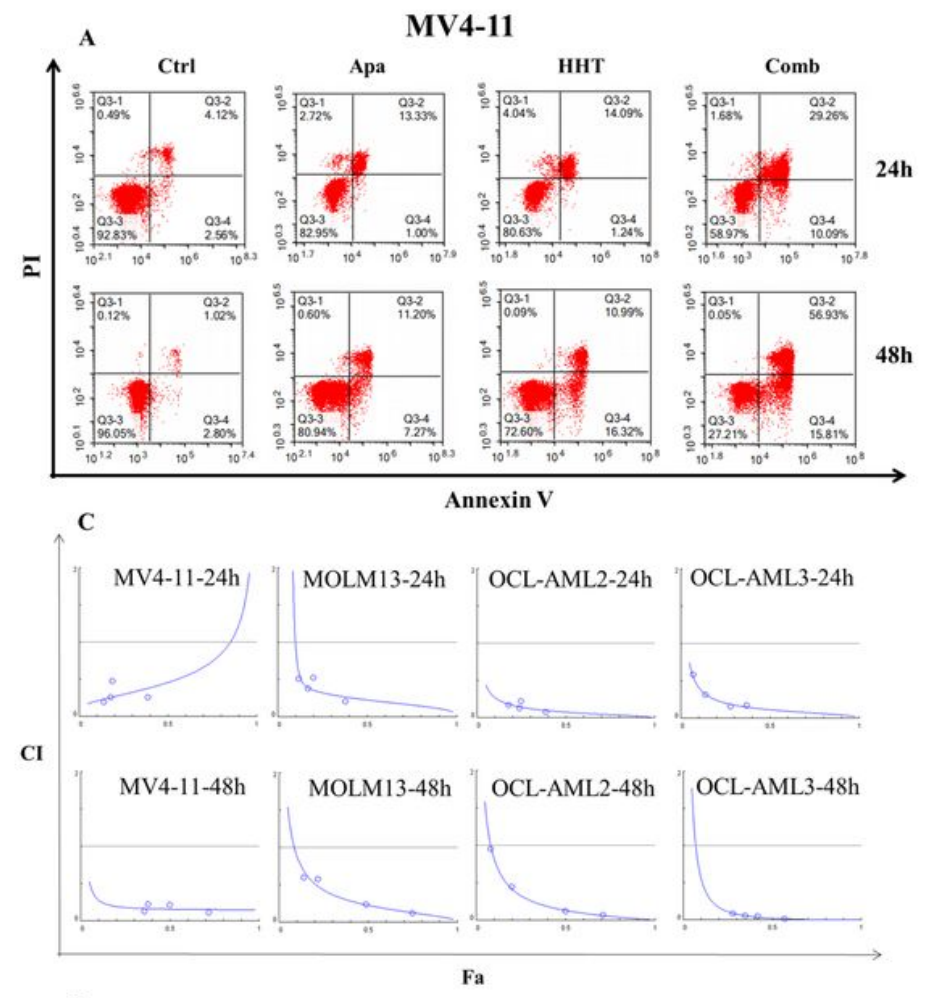

THP1-24h

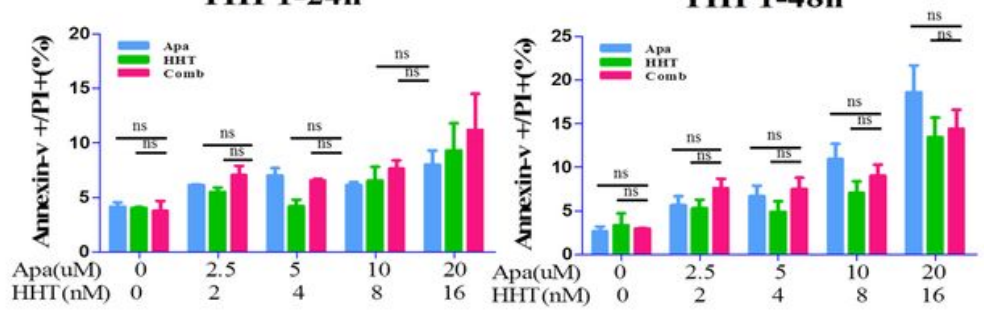

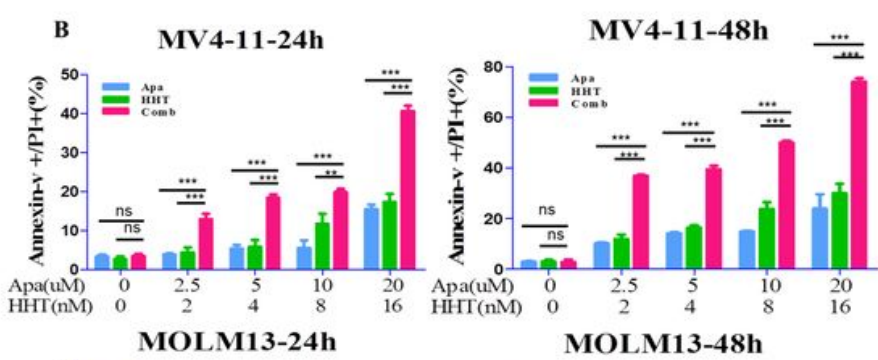

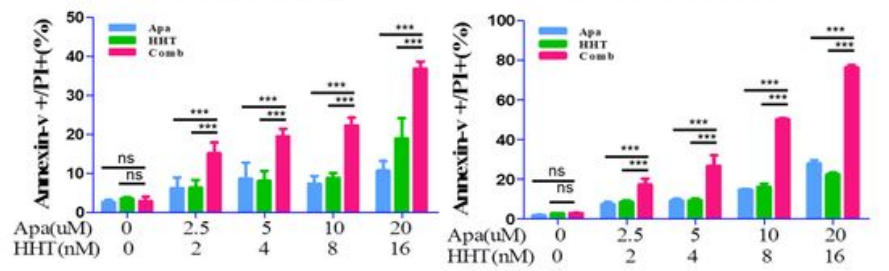

OCI-AML2-24h

OCI-AML2-48h

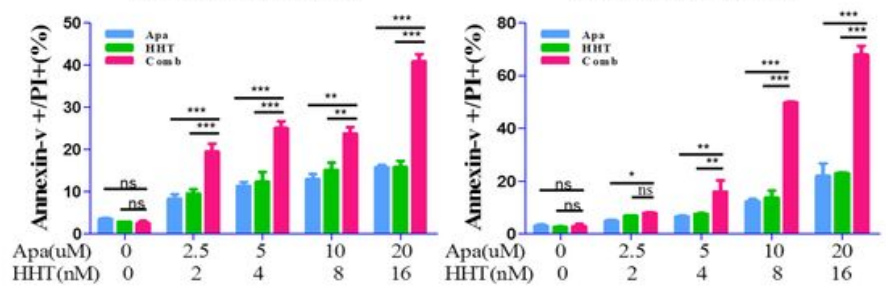

OCI-AML3-48h

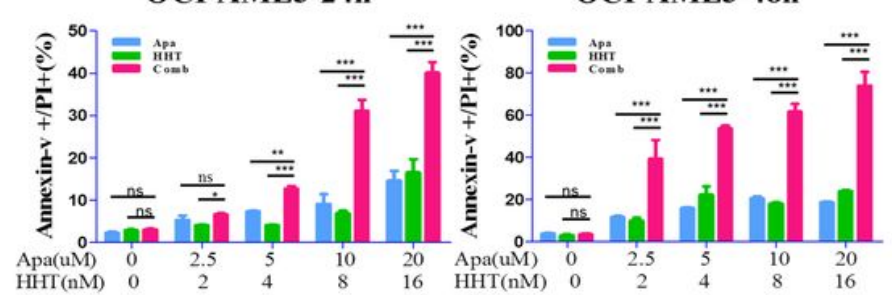

Figure 2

The percentage of apoptotic cells was examined with a NovoCyte flow cytometer (A). Apoptosis induced by various treatments at $48 \mathrm{~h}$ Apatinib combined with HHT resulted in significant increases in the apoptosis rate in AML cell lines $(B)$. Combination index $(\mathrm{CI})$ values were calculated according to the median effect method of Chou and Talala (C) and Table 2. The combination of Apatinib and HHT displayed no effect on the THP1 cell line without FLT3-ITD mutations (D). ${ }^{*} p<0.05 ;{ }^{* \star} p<0.01 ;{ }^{\star \star *} p<$ 0.001 . 

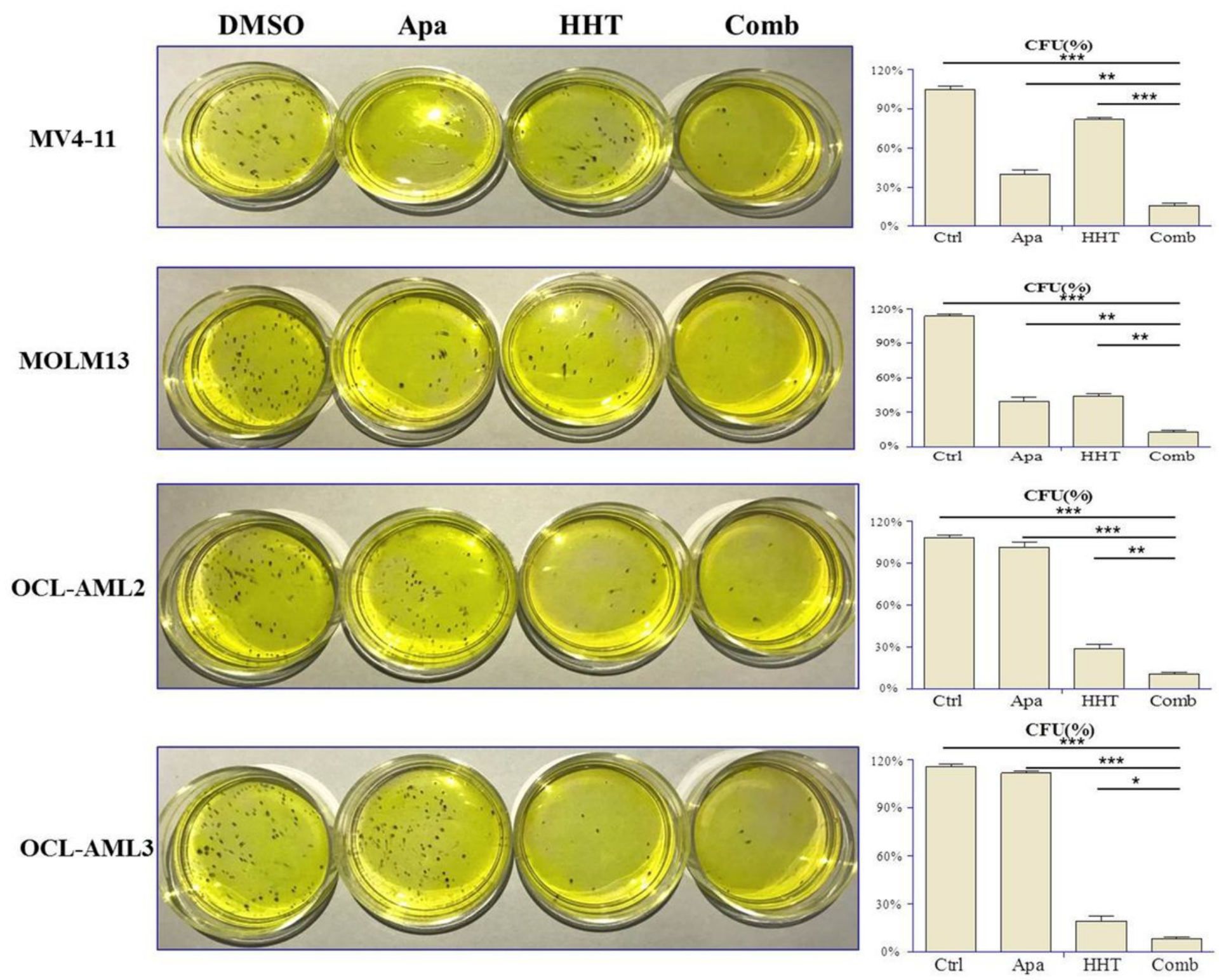

\section{Figure 3}

MV4-11, MOLM-13, OCL-AML2, OCL-AML3 cells (2×105/well) were used to test colony-forming abilities. AML cells were seeded in 24-well plates and then, treated with $20 \mu \mathrm{M}$ Apatinib or $16 \mathrm{nM}$ HHT alone or both molecules. After $24 \mathrm{~h}$, cells were washed and then cultured in complete methylcellulose medium at a cell density of 500 cells/well in 3.5-cm dishes for 10-14 days. The percentage of CFU was determined by counting colonies ( $\geq 50$ cells). Data are presented as the mean \pm S.D. of three independent experiments. ${ }^{*} \mathrm{p}<0.05 ;{ }^{* *} \mathrm{p}<0.01$; *** $\mathrm{p}<0.001$. 
A

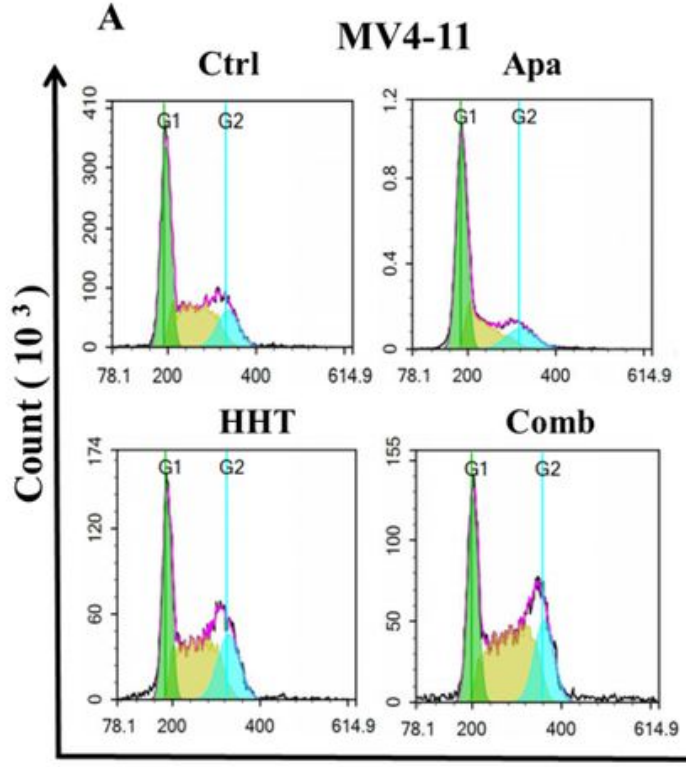

MV4-11-24h

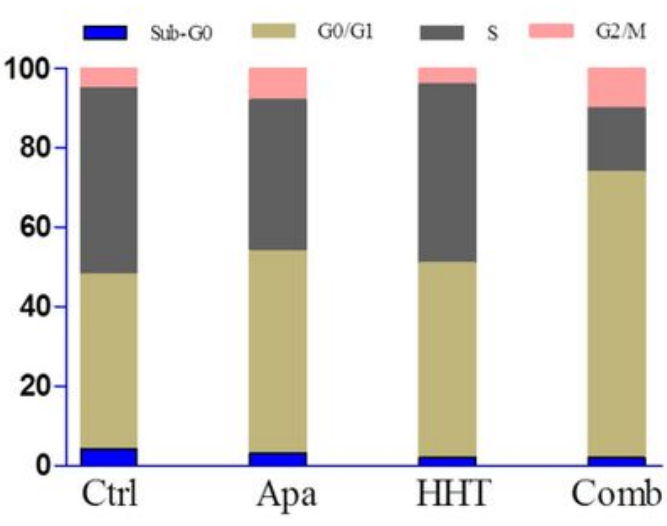

B

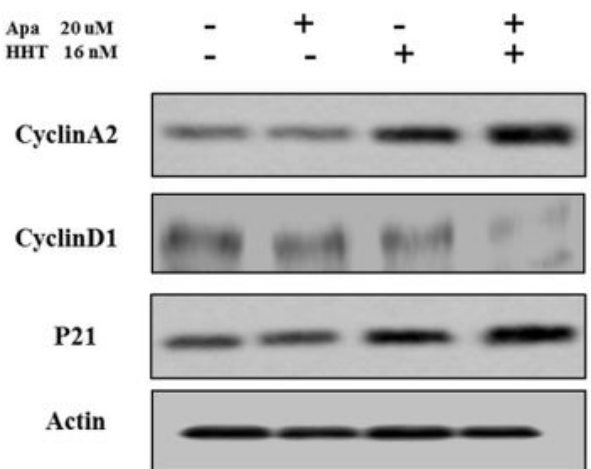

MOLM13
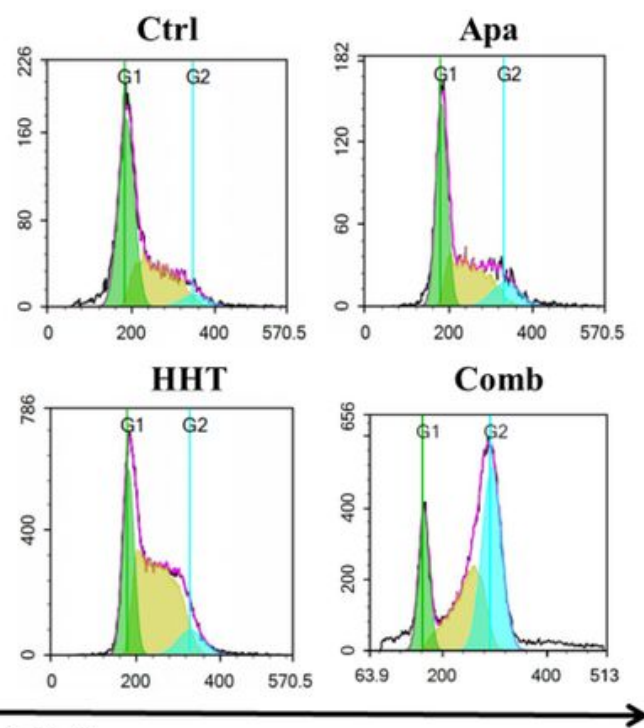

PE-H $\left(10^{3}\right)$

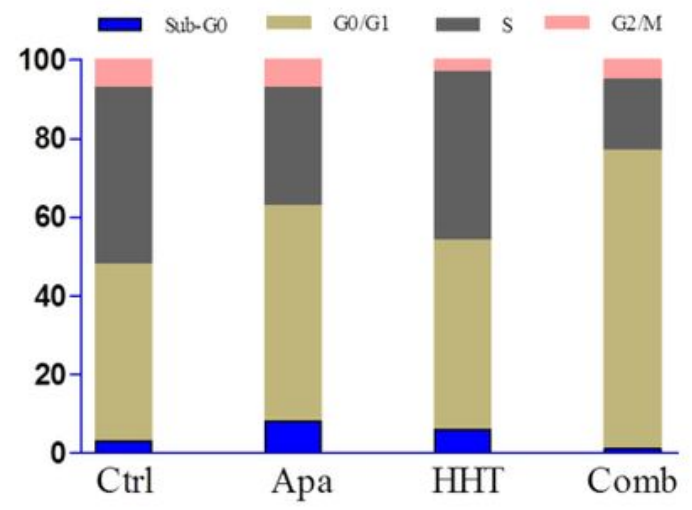

\section{Figure 4}

In MV4-11 and MOLM13 cells, G0/G1 phase arrest and S phase decreased were observed after treatment with Apatinib and HHT. (A) After drug treatment for $24 \mathrm{~h}$, cell cycle distribution was analyzed using flow cytometry ( ${ }^{*} \mathrm{P}<0.05$, One-way ANOVA, combination treatments versus control, and single treatments). (B) Western blot analysis showed an increase in cell cycle regulator cyclinA2, cyclinD1, and p21 in cells treated with Apatinib and HHT compared to a single agent. Actin served as a loading control. 


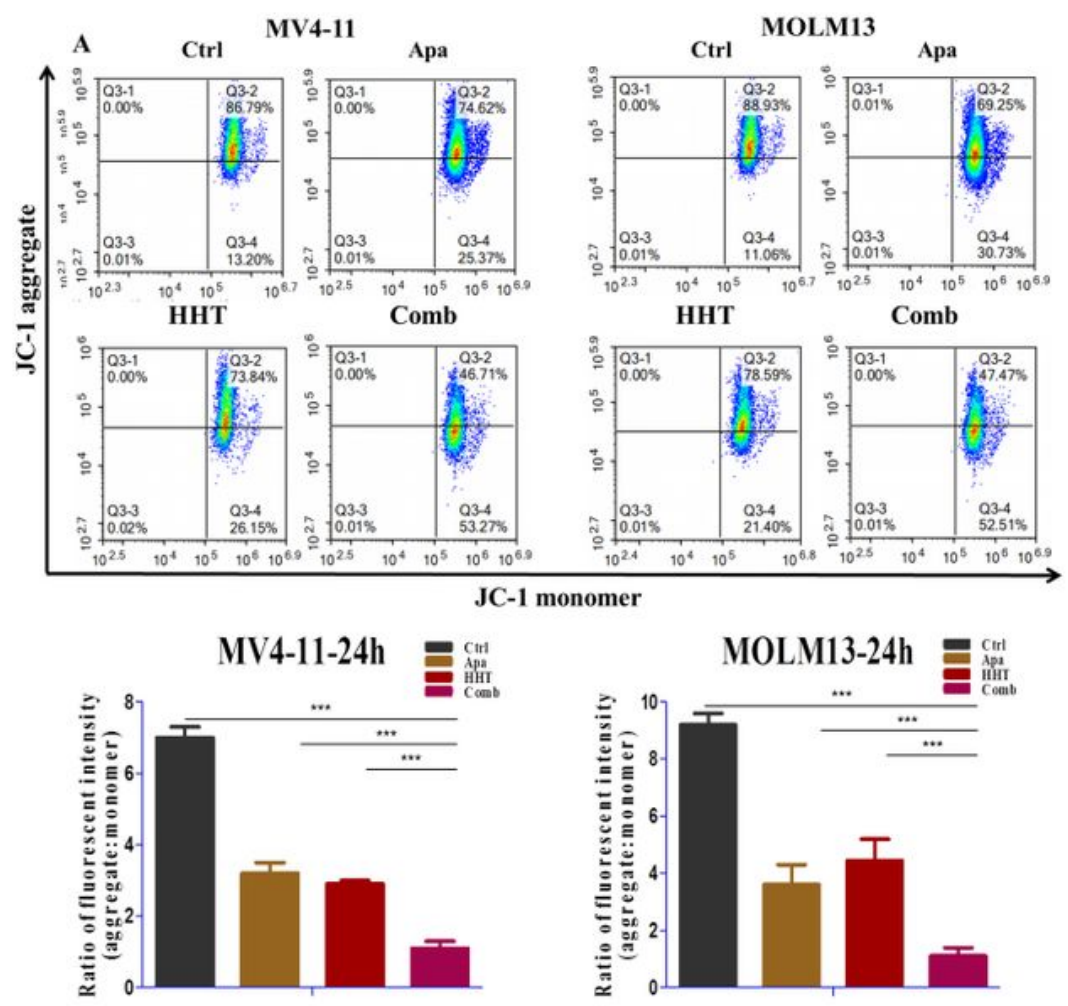

B

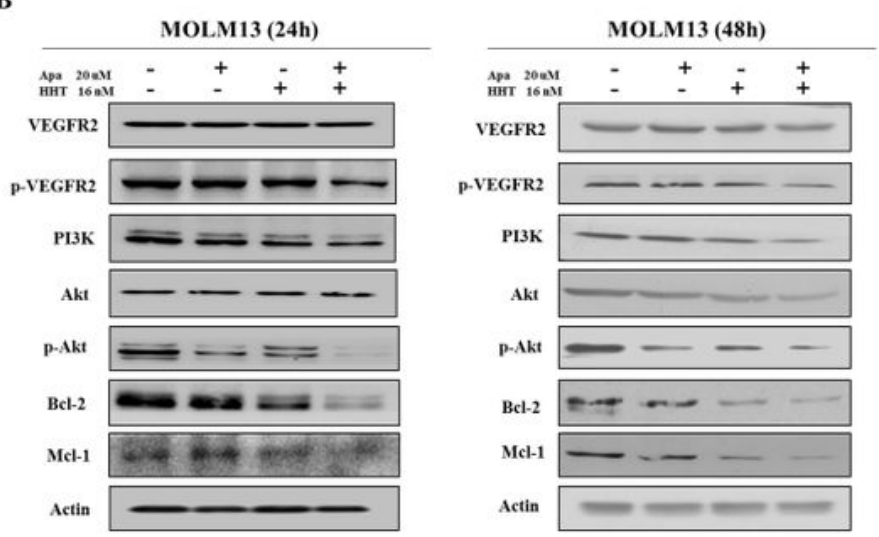

C

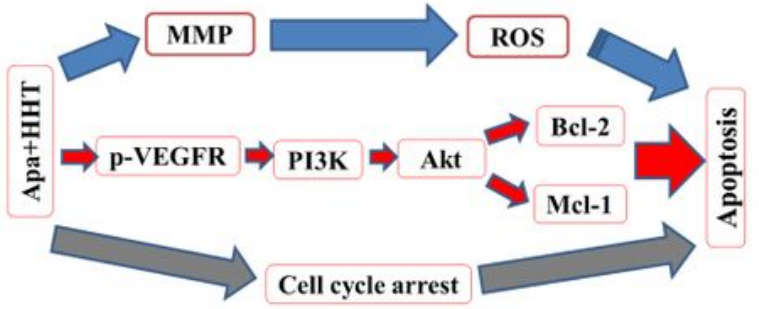

\section{Figure 5}

In the MV4-11 and MOLM-13 cell lines, first we used JC1 kit to detect the influence of different concentrations of Apatinib and HHT on mitochondrial membrane potential, the results showed that the combination group significantly reduced the mitochondrial membrane potential (A). Besides, Western blot results showed that Apatinib combined with HHT downregulated p-VEGFR2 expression. Strikingly, our results also showed that cotreatment with Apatinib and HHT inhibited the PI3K, Akt signaling cascade, 
and the anti-apoptosis protein levels of $\mathrm{Bcl}-2$ and $\mathrm{Mcl}-1$ were determined by Western blotting (B). An FLT3ITD mutation AML cells Apatinib combined with HHT induced cell apoptosis by decreasing mitochondrial membrane potential, inhibiting the cell cycle, and regulating vascular endothelial growth factor as well as its downstream signaling pathways (C).

\section{Supplementary Files}

This is a list of supplementary files associated with this preprint. Click to download.

- Table1.doc

- Table2.doc 